\title{
Genetic and metabolic engineering
}

\author{
Yea-Tyng Yang \\ Department of Bioengineering and Chemical Engineering Rice University, P.O. Box 1892, Houston, Texas 77251-1892 \\ Tel :(713) 527-8101 Fax:(713)737-5877 \\ E-mail: tinayang@ rice.edu \\ George N. Bennett \\ Department of Biochemistry and Cell Biology Rice University, P.O. Box 1892, Houston, Texas 77251-1892 \\ Tel:(713) 527-8101 Fax:(713)737-5877 \\ E-mail: gbennett@bioc.rice.edu \\ Ka-Yiu San ${ }^{1}$ \\ Department of Bioengineering and Chemical Engineering Rice University, P.O. Box 1892, Houston, Texas 77251-1892 \\ Tel :(713) 527-8101 Fax:(713)737-5877 \\ E-mail : ksan@rice.edu \\ http://www.rice.edu
}

\begin{abstract}
Recent advances in molecular biology techniques, analytical methods and mathematical tools have led to a growing interest in using metabolic engineering to redirect metabolic fluxes for industrial and medical purposes. Metabolic engineering is referred to as the directed improvement of cellular properties through the modification of specific biochemical reactions or the introduction of new ones, with the use of recombinant DNA technology (Stephanopoulos, 1999). This multidisciplinary field draws principles from chemical engineering, biochemistry, molecular and cell biology, and computational sciences. The aim of this article is to give an overview of the various strategies and tools available for metabolic engineers and to review some of the recent work that has been conducted in our laboratories in the metabolic engineering area.
\end{abstract}

Metabolic engineering is generally referred to as the targeted and purposeful alteration of metabolic pathways found in an organism in order to better understand and utilize cellular pathways for chemical transformation, energy transduction, and supramolecular assembly (Lessard, 1996). This multidisciplinary field draws principles from chemical engineering, computational sciences, biochemistry, and molecular biology. In essence, metabolic engineering is the application of engineering principles of design and analysis to the metabolic pathways in order to achieve a particular goal. This goal may be to increase process productivity, as in the case in production of antibiotics, biosynthetic precursors or polymers, or to extend metabolic capability by the addition of extrinsic activities for chemical production or degradation. Previous strategies to attain these goals seem more of an art with experimentation by trial-and-error. Two papers in the early 1990s advocated the switch from this artistic approach to a more systematic and rational approach (Bailey, 1991, Stephanopoulos and Vanillo, 1991). This scientific approach would involve the use of recombinant DNA technology and a better understanding of cellular physiology to modify intermediary metabolism.

Several reviews on metabolic engineering cover general (Cameron and Tong, 1993, Stephanopoulos and Sinskey, 1993, Farmer and Liao, 1996, Cameron and Chaplen, 1997) and specific areas (for example, yeast: Hansen and Kiellandbrandt, 1996; plant: Dixon and Arntzen, 1997, Cunningham and Gantt, 1998; Escherichia coli: Berry, 1996). The overview by Cameron and Tong (1993) presents a thorough description of early metabolic engineering endeavors. Also of special interest is the issue of Biotechnology and Bioengineering (Vol. 58, 1998) dedicated solely to the topic of metabolic engineering.

The interest in metabolic engineering is stimulated by potential commercial applications where improved methods for developing strains which can increase production of useful metabolites. Recent endeavors have focused on using biologically derived processes as alternatives to chemical processes. Such manufacturing processes pursue goals related to "sustainable developments" and "green chemistry" as well as positioning companies to exploit advances in the biotechnology field. Some examples of these new processes include the microbial production of indigo (Genencor) and propylene glycol (DuPont) and others involve improvements in the more traditional areas of antibiotic, and amino acid production by a large number of firms. The extension of metabolic engineering to production of desired compounds in plant tissues and to provide better

\footnotetext{
${ }^{1}$ Corresponding author
} 
understanding of genetically determined human metabolic disorders broadens the interest in this field beyond the fermentation industry and bodes well for its future importance.

\section{Engineering Strategies}

\section{Physiological studies}

The concept of network rigidity, flexible and rigid nodes, was introduced by Stephanopoulos and Vallino (1991). The rigidity of a network or its resistance to variations in metabolic change is due to control mechanisms established to ensure balanced growth. For a engineering strategy to be successful, a better understanding of the host cell is necessary to determine the types of genetic modifications needed to achieve the final goal. Some of the physiological considerations that should be examined include the effects of genetic manipulation on growth and possible effects on "unrelated" systems. These negative effects of genetic manipulation are often attributed to the metabolic burden. In the case of the over-expression of phosphoenol pyruvate forming enzymes, the heat-shock response and nitrogen regulation were both inhibited (Liao et al., 1996a).

\section{Selection of the method of genetic modification}

In terms of DNA techniques, several approaches have been used for the modification of the host cell to achieve the desired goal. These include: the removal of the enzyme or its inhibition to eliminate a competitive pathway (Green et al., 1996; Gasson et al., 1996; Shimada et al., 1998) or a toxic byproduct (Aristidou et al., 1994; Cameron et al., 1998, Chaplen at al., 1996); the amplification of a gene or group of genes to improve the synthesis of existing products (Pines et al., 1997; Shimada et al., 1998; Lu and Liao; 1997); the expression of an heterologous enzyme(s) to extend the substrate range (Prieto et al., 1996; Panke et al., 1998; Sprenger, 1996), to produce novel product (Chopra and Vageeshbabu, 1996; Hershberger, 1996; Ingram et al., 1998; Misawa and Shimada, 1998; Poirier et al., 1995; Stassi et al., 1998), to provide pathways for the degradation of toxic compounds (Chen and Wilson, 1997; Keasling et al., 1998; Xu et al., 1996), or to design a more environmentally resistant plant (Smirnoff, 1998). Alternatively, the deregulation of existing enzymes might be necessary to overcome the existing control mechanisms of a rigid node. A combination of modifications may be needed to achieve the goal. Additional manipulation of the central metabolic pathway may also be required to generate the precursor, cofactors, and energy needed to sustain the modified pathway (Stephanopoulos and Sinskey, 1993, Farmer and Liao, 1996). The manipulation of central metabolism has been exemplified in the production of aromatic compounds in E. coli growing in glucose (Berry, 1996).

\section{Inverse metabolic engineering}

The classical approach of metabolic engineering, as discussed above, requires detailed knowledge of the enzyme kinetics, the system network, and intermediate pools involved, and on such bases, a genetic manipulation is proposed for some presumed benefits. In contrast, the concept of inverse metabolic engineering is first to identify the desired phenotype, then to determine environmental or genetic conditions that confer this phenotype, and finally to alter the phenotype of the selected host by genetic manipulation (Bailey et al., 1996, Delgado and Liao, 1997). As in the case of expression of the oxygen binding protein, $\mathrm{VHb}$, in E. coli, the observed phenotype of high heme cofactor levels in an obligate aerobe Vitreoscilla under oxygen limitation suggested that synthesis of the hemoglobin could improve growth of other organisms under similar limitations (Bailey et al., 1996).

\section{Mathematical Tools for Analysis}

\section{Metabolic flux analysis}

Metabolic flux analysis (MFA) is based on a known biochemistry framework. A linearly independent metabolic matrix is constructed based on the law of mass conservation and on the pseudo-steady state hypothesis (PSSH) on the intracellular metabolites. The formulation resulted in a set of linear equations that can be expressed as a stoichiometric matrix $\mathbf{A}$ of dimension $\mathrm{m}$ by $\mathrm{n}$ with vectors for net accumulation, $\mathrm{r}(\mathrm{mx} 1)$, and metabolic flux, $v(\mathrm{nx} 1)$. No kinetic data is required. Dynamic fluxbalance analysis can be obtained from the same derivation as shown in the following equation with $\mathbf{b}$ as the transport term.

$$
\mathbf{r}=\frac{\mathrm{d} \mathbf{X}}{\mathrm{dt}}=\mathbf{A} \cdot \mathbf{v}-\mathbf{b}
$$

Typically, the system that results is under-determined system where $\mathrm{m}>\mathrm{n}$. However under certain conditions, some pathways are inoperative and can be neglected. The system may become completely determined or overdetermined and can be solved along with the measurements of external or internal fluxes (Delgado and Liao, 1997, Chen et al., 1997). Non-invasive methods of analysis such as nuclear magnetic resonance (NMR) can also provide information on the structure of the biochemical network as well as flux measurements (Alam and Clark, 1989; Follstad and Stephanopoulos, 1998; Park et al., 1997; Schmidt et al., 1998). Holms (1996) has calculated the metabolic fluxes of $E$. coli on various carbon substrates and this serves as a useful database. 


\section{Metabolic Control Theory (MCT)}

Metabolic Control Theory was independently developed by Kacser and Burns (Kacser and Burns, 1973) and Heinrich et al. (1997) to identify the kinetic constraints in a biochemical network. A similar approach, that of Biochemical Systems Theory (BST), was developed by Savageau et al. (1987a; 1987b). A more structural approach to MCT has been developed (Fell, 1992). Based upon the kinetic data, the control coefficients can be calculated. This approach is used to determine the ratelimiting reaction in a network. However, a single ratelimiting step may not exist and several steps may share the control of the metabolic network. Three commonly used normalized sensitivity measures, which quantify how the control of steady state fluxes and concentrations is distributed between different reactions in a metabolic network, have been defined as:

Flux Control Coefficient (FCC):

$$
\mathrm{C}_{\mathrm{E}}^{\mathrm{J}}=\frac{\mathrm{dJ}}{\mathrm{dE}} \frac{\mathrm{E}}{\mathrm{J}}
$$

Concentration Control Coefficient (CCC):

$$
\mathrm{C}_{\mathrm{E}}^{\mathrm{X}}=\frac{\mathrm{dX}}{\mathrm{dE}} \frac{\mathrm{E}}{\mathrm{X}}
$$

$$
\begin{aligned}
& \text { Elasticity: } \\
& \varepsilon_{\mathrm{X}}^{\mathrm{V}_{\mathrm{A}}}=\frac{\partial \mathrm{v}_{\mathrm{A}}}{\partial \mathrm{X}} \frac{\mathrm{X}}{\mathrm{v}_{\mathrm{A}}}
\end{aligned}
$$

The flux control coefficient is a measure of how a change in the concentration of the enzyme $\mathrm{E}$ affects the steadystate flux through that particular enzyme. That is, it is measure of the degree of control exerted by enzyme $E$ on this steady-state flux. The concentration control coefficient is a measure of the extent of control exerted by the enzyme $\mathrm{E}$ on the steady-state concentration $\mathrm{X}$, while the elasticity is a measure of the response of the reaction rate upon changes in the concentration $\mathrm{X}$.

The extension of MCT to be applicable under large changes in enzyme activities and effector concentration has recently been published (Small and Cacser, 1993a, 1993b). This extension would allow for experimental calculations of the deviation index from MFA data. Several articles dealing with various aspects of metabolic control analysis have also appeared recently (Ehlde and Zacchi, 1997; Mauch et al., 1997; Stephanopoulos and Simpson, 1997).

A top-down approach for the determination of control coefficients was developed by Brown et al. (1990), which allows for the determination of control coefficients based on relative flux alone and avoids the need for values of enzyme activities. In addition, more information is obtained from a single application of the top-down method. There are certain limitations to this method. The method is invalid if more than one kinetic link exist between the two ends of a pathway (i.e., more than one enzyme catalyzes the same reaction). In addition, an small overall flux control coefficient(FCC) does not necessarily mean the individual FCCs are small (Brown et al., 1990). However, it can be used in certain limited situations (Brown et al., 1990).

\section{An Example of Metabolic Engineering}

\section{Metabolic engineering of the central metabolic pathways of E. COli - Strategies for acetate reduction to achieve high recombinant protein production}

In the past several years the work in our laboratories has been focusing on the various aspects of manipulating the central metabolic pathways in E. coli Specifically, we are interested in the effect of precise genetic manipulations on the metabolic activities of E. COli A potential application is to address the problem of acetate accumulation in an $\mathrm{E}$. coli culture. One of the major technical challenges in recombinant protein production processes is to achieve high expression level of the cloned gene and high cell density. Unfortunately, under these demanding conditions, the amount of acetate accumulated in the reactor increases enormously, often to a level that has a detrimental effect on both cell and protein yields.

Various operational strategies have been proposed and tested in the past to reduce the extent of acetate accumulation (Chen and McDonald, 1990; Curless et al., 1991). Most of these approaches fall into one of the following three major categories: 1) avoidance of dissolved oxygen limitation by increasing the agitation speed or enrichment with pure oxygen; 2) restricting the quantity of nutrient available for cell growth by adjusting the medium feed rate using sophisticated control algorithms during fedbatch operation; 3) removal of toxic wastes, especially acetate from spent medium, from the reactor using in situ devices, such as a perfusion system. Although these strategies can lead to an improved process performance, their implementation is very difficult in practice since the operating window to achieve optimal results requires extremely tight control as well as being expensive to implement.

It is our goal to reduce the level of acetate accumulation through the manipulation of cellular activities. Figure 1 depicts a simplified scheme of glucose metabolic pathways of E. Coli One of the major pathways leading to the formation of acetate is also shown. Several potential points of attack leading to a lower rate of acetate production can 
be envisioned upon close inspection of the pathways involved. One obvious approach is the destruction or complete elimination of the portion of the reaction pathway leading to acetate formation at the acetyl-CoA node. Other approach include a better regulation of carbon source uptake and therefore reducing the accumulation of excessive pyruvate or acetyl-CoA. Another possibility is the creation of a new reaction pathway that will fit into the existing glycolysis pathways by introducing foreign genes encoding an appropriately chosen enzyme system(s). It is argued that a properly designed new reaction pathway will perturb the existing pathway networks positively and will result in a better strain with the desired cellular characteristics

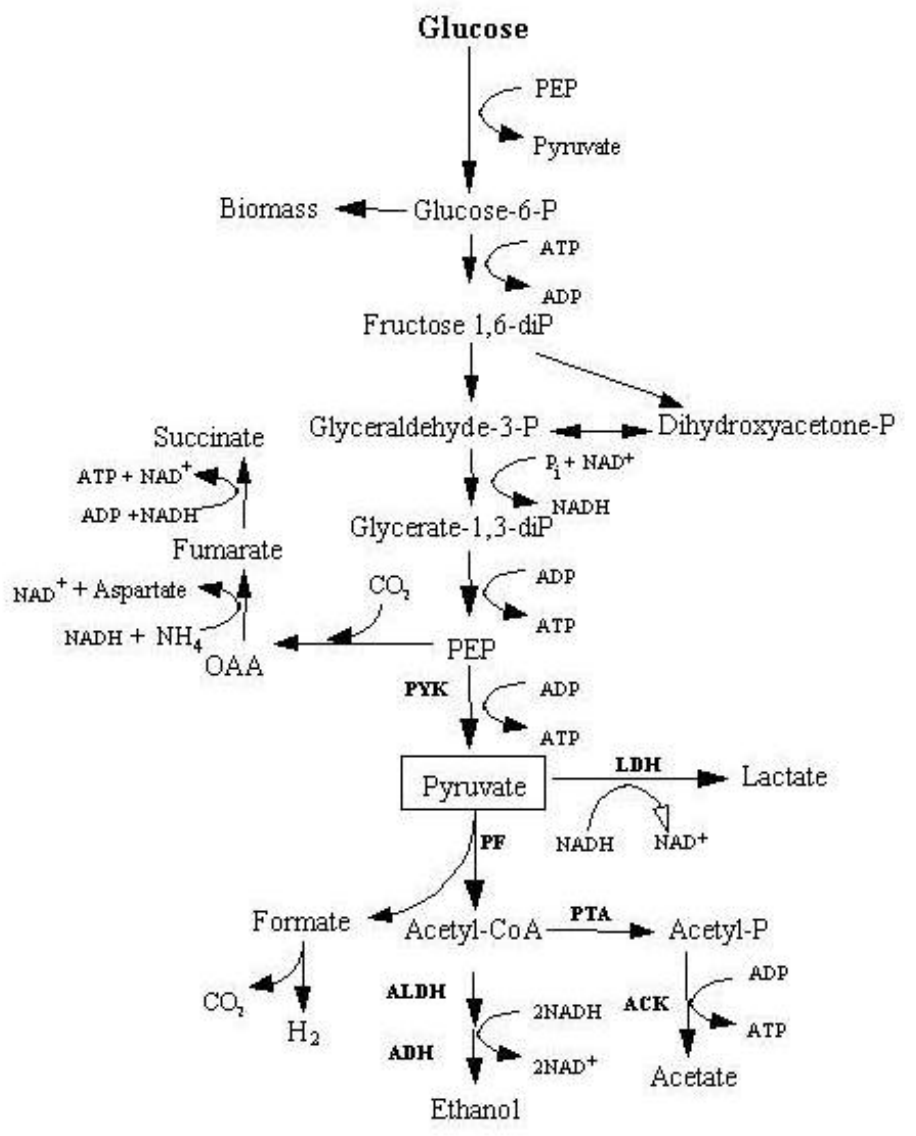

Figure 1. Schematic of the central metabolic pathways of E. coli.

Two different approaches have been investigated to modulate the glucose uptake rate in E. coli (Chou et al., 1994a; Chou et al., 1994b). In the first study, methyl $\alpha$ glucoside $(\alpha-\mathrm{MG})$, a glucose analog, which shares the same phosphotransferase system (PTS) was used to modulate the glucose uptake rate. This compound is a nontoxic, metabolically inert, competitive inhibitor to enzyme $\mathrm{II}^{\mathrm{glc}}$ of the PTS. The glucose analog addition resulted in a reduced acetate accumulation in the reactor and subsequently led to a significant enhancement in recombinant protein production (Chou et al., 1994a). Based on the results from this study, a metabolically engineered $E$ coli strain with reduced acetate synthesis rate was constructed through the modification of glucose uptake rate (Chou et al., 1994b). It is argued that glucose uptake is not well regulated in $E$. coli and that an imbalance between catabolism and anabolism can result in excess carbon flux; the cell subsequently shunts this excess flux through the acetate pathway. It is further argued that the modification of $p t s G$, which encodes the PEP: glucose phosphotransferase system, will reduce glucose uptake and thus will lower the acetate synthesis rate. An E. coli strain bearing a mutation in pts $\mathrm{G}$ was thus constructed and characterized. It was shown that the growth rate of the mutant strain was slower than its parent in glucose defined medium but was not affected in complex medium. Furthermore, experimental results using this mutant strain showed significant improvement in culture performance due to reduced acetate excretion. Both biomass and recombinant protein production, using $\beta$-galactosidase as a 
model protein, increased by more than $50 \%$. Recombinant protein productivity at a level of more than $1.6 \mathrm{~g} / \mathrm{l}$ using this mutant strain was reported (Chou et al., 1994b).

The redirection of carbon flux toward less inhibitory byproducts was also investigated. This approach for acetate reduction involved the heterologous expression of the Bacillus subtilis acetolactate synthase (ALS) gene in E. coli (Aristidou et al., 1994, Aristidou et al., 1995); the ALS enzyme converts pyruvate to acetoin. The choice of this particular gene was based on the low acetate production pattern of organisms that naturally contain this enzyme. The pyruvate branchpoint was chosen as the point of flux redirection due to its central position in the metabolic network. The critical node in this case is thus the pyruvate branch point where carbon flux partitioning between lactate, acetyl-CoA and acetoin occurs. Figure 2 shows a modified schematic diagram, which includes the newly added reaction pathway. The heterologous expression of the ALS catabolic enzyme in E. coli drastically modified the cellular glycolytic fluxes. Specifically, acetate excretion into the broth was minimized and always maintained at a level well below the toxic threshold. It was also shown that acetoin, the byproduct from the newly added pathway, was about 50 times less harmful than acetate. Moreover, the expression of the biologically active ALS enzyme in E. coli has been shown to have a negligible effect on cell physiology; both specific growth rate and cell yields were not affected (Aristidou et al, 1994). Chemostat experiments and subsequent metabolic flux analysis further revealed that the ALS bearing cells were capable of maintaining a reduced specific acetate production rate at dilution rates ranging from 0.1 to $0.4 \mathrm{hr}^{-1}$. In addition, the ALS strain was shown to have a higher ATP yield and lower maintenance coefficient (Aristidou et al., 1998). Using this genetically engineered strain as the host for recombinant protein production led to a significant increase in culture performance. The expression of a model recombinant $\mathrm{CadA} / \beta$-galactosidase fusion protein under the control of a strong $\mathrm{pH}$-regulated promoter showed an increase of about $60 \%$ for the specific protein activity (to a level of $30 \%$ of total cellular protein) and $50 \%$ in terms of the volumetric activity in batch cultures (Aristidou et al., 1995).

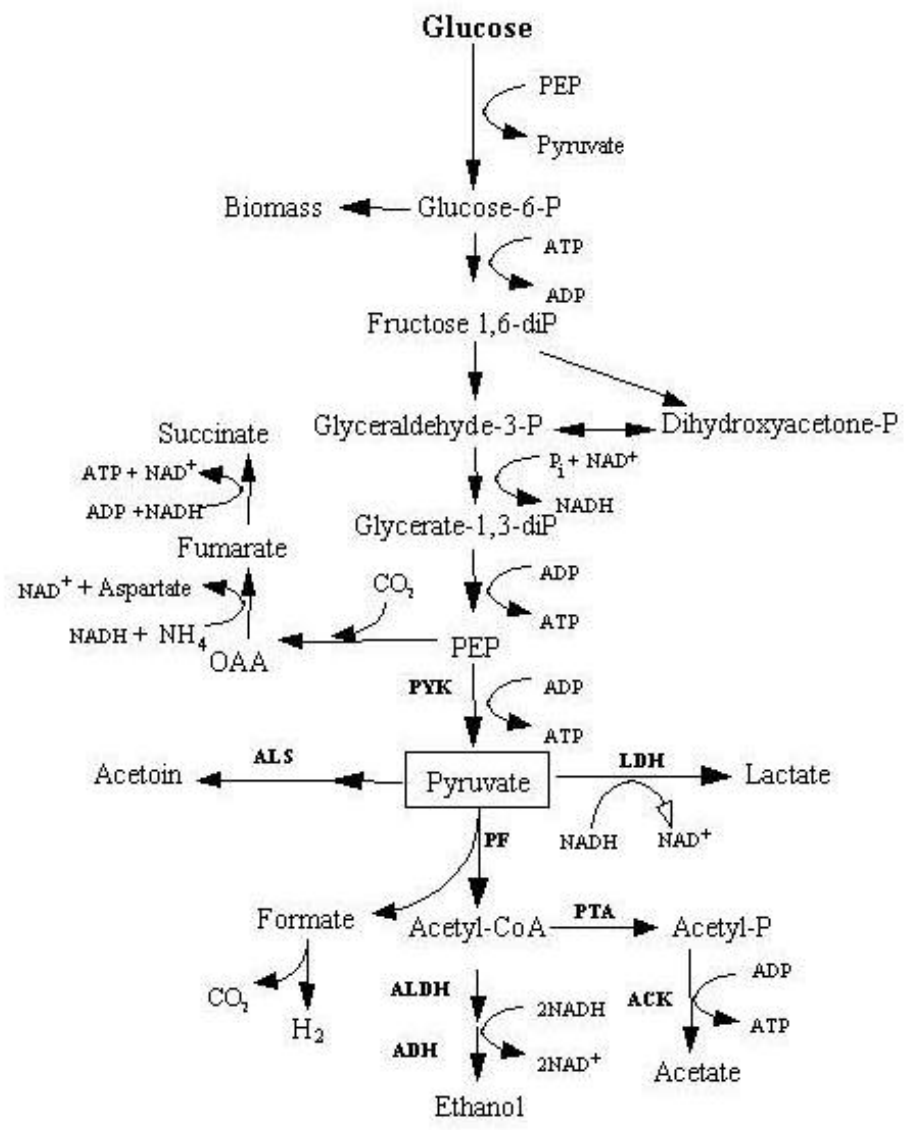

Figure 2. Modified schematic of the central metabolic pathways of E. coli to include the newly added ALS pathway. 
The final examples are related to the manipulation of pathways at the pyruvate and acetyl-CoA nodes. In particular, we have investigated the effect of the elimination of critical enzymes that are involved directly in the formation of acetate, specifically the acetate kinase phosphotransacetylase (ack-pta) pathway, on cellular behavior and culture performance (San et al., 1994; Yang et al., 1998). The destruction of this pathway resulted in low acetate levels at the expense of cell fitness. The mutant strain had a much-lowered specific ethanol synthesis rate; excess carbon flux was channeled through the lactate synthesis pathway instead (Yang et al., 1998). In other words, the LDH pathway from pyruvate becomes much more competitive in this ack-pta mutant strain. In another related study, reduction of acetate accumulation through the isolation of mutant strains that are deficient in one of the major acetate synthesis pathways was examined (San et al., 1995). The behavior and performance of two isolated fluoroacetate resistant mutants were then evaluated with different medium composition under various growth environments. Particularly, the performance of the fluoroacetate resistant mutants under strong recombinant gene expression and relatively high cell density was studied using a strong $\mathrm{pH}$-inducible promoter system. It was reported that the fluoroacetate-resistant mutant AAA-1 ( $\left.a c k^{-}\right)$consistently out-performed its parent strain, GJT-1. The AAA-1 strain accumulated less acetate and produced significantly more recombinant protein. Using a previously reported $\mathrm{pH}$-inducible promoter system, the production of $\beta$-galactosidase at a level as high as $40 \%$ of the total cellular soluble proteins while achieving an optical density higher than 25 could be routinely obtained. In addition, the performance of the AAA-1 mutant was found to be less sensitive to the reactor dissolved oxygen level (San et al., 1995).

\section{References}

Alam, K.Y. and Clark, D. P. (1989). Anaerobic fermentation balance of Escherichia coli as observed by in vivo nuclear magnetic resonance spectroscopy. Journal of Bacteriology 171: 6213-6217.

Aristidou, A.A., San, K.Y. and Bennett, G.N. (1994). Modification of central metabolic pathway in Escherichia coli to reduce acetate accumulation by heterologous expression of the Bacillus subtilis acetolactate synthase gene. Biotechnology \& Bioengineering 44:944-951.

Aristidou, A. A., Bennett, G.N. and San, K.Y. (1995). Metabolic engineering of Escherichia coli to enhance recombinant protein production through acetate reduction. Biotechnology Progress 475-478.

Aristidou, A. A., Bennett, G.N., and San, K.Y. (1998). Metabolic flux analysis of Escherichia coli expressing the
Bacillus subtilis acetolactate synthase in batch and continuous cultures. Submitted.

Bailey, J. E. (1991). Toward a science of metabolic engineering. Science 252:1668-1675.

Bailey, J. E., Shurlati, A., Hatzimanikatis, V., Lee, K., Renner, W.A.and Tsai, P.E. (1996). Inverse metabolic engineering a strategy for directed genetic engineering of useful phenotypes. Biotechnology \& Bioengineering 52:109-121.

Berry, A. (1996). Improving production of aromatic compounds in Escherichia coli by metabolic engineering. Trends in Biotechnology 14:219-259.

Brown, G.C., Hafner, R.P. and Brand, M.D. (1990). A 'top-down' approach to the determination of control coefficients in metabolic control theory. European Journal of Biochemistry 188:321-325.

Cameron, D.C., Altaras, N.E., Hoffman, M.L. and Shaw, A.J. (1998). Metabolic engineering of propanediol pathways. Advances in Biochemical Engineering 14:116125.

Cameron, D.C. and Chaplen, F. (1997). Developments in metabolic engineering. Current Opinions in Biotechnology 8:175-180.

Cameron, D.C. and Tong, I.T. (1993). Cellular and metabolic engineering. Applied Biochemistry and Biotechnology 38:105-140.

Chaplen, F. W. R., Fahl, W.E. and Cameron, D.C. (1996). Effect of endogenous methylglyoxal on chinese hamster ovary cell grown in culture. Cytotechnology 22:33-42.

Chen, C.-I., and McDonald, K.A. (1990). Oscillatory behavior of Saccharomyces cerevisiae in continuous culture: I. Effect of $\mathrm{pH}$ and nitrogen levels. Biotechnology \& Bioengineering 36:19-27.

Chen, R., Hatzimanikatis, V., Yap, W., Postma, P.W. and Bailey, J.E. (1997). Metabolic consequences of Phosphotransferase (PTS) mutation in a phenylalanineproducing recombinant Escherichia coli. Biotechnology Progress 13:768-775.

Chen, S.L.and Wilson, D.B. (1997). Genetic engineering of bacteria and their potential for $\mathrm{Hg}^{2+}$ bioremediation. Biodegradation 8:97-103.

Chopra V.L. and Vageeshbabu H.S. (1996). Metabolic engineering of plant lipids. Journal of Plant. Biochemistry \& Biotechnology 5:63-68. 
Chou, C.-H., Bennett, G. N. and San, K.Y. (1994a). Effect of modulated glucose uptake on high-level recombinant protein production in a dense Escherichia coli culture. Biotechnology Progress 10:644-647.

Chou, C.-H., Bennett, G. N. and San, K.Y. (1994b). Effect of modified glucose uptake using genetic engineering techniques on high-level recombinant protein production in Escherichia coli dense cultures. Biotechnology \& Bioengineering 44:952-960.

Cunningham, F.X. and Gantt, E. (1998). Genes and enzymes of carotenoid biosynthesis in plants. Molecular Biology 49:557-583.

Curless, C., Fu, K., Swank, R., Menjares, A., Fieschko, J., and Tsai, L. (1991). Design and evaluation of a two-stage, cyclic, recombinant fermentation process. Biotechnology \& Bioengineering 38:1082-1090.

Delgado, J. and Liao, J.C. (1997). Inverse flux analysis for reduction of acetate excretion in Escherichia coli. Biotechnology Progress 13:361-367.

Dixon, R.A. and Arntzen, C.J. (1997). Transgenic plant technology is entering the era of metabolic engineering. Trends in Biotechnology 11:441-444.

Ehlde, M.and Zacchi, G. (1997). A general formalism for metabolic control analysis. Chemical Engineering Science 52:2599-2606.

Farmer, W.R. and Liao, J.C. (1996). Progress in metabolic engineering. Current Opinion in Biotechnology 7:198-204.

Fell, D.A. (1992). Metabolic control analysis: a survey of its theoretical and experimental development. Biochemical Journal 286:313-330.

Follstad, B. D. and Stephanopoulos, G. (1998). Effect of reversible reactions on isotope label redistribution analysis of the pentose phosphate pathway. European Journal of Biochemistry 252: 360-371.

Gasson, M. J., Benson, K., Swindell, S. and Griffin, H. (1996). Metabolic engineering of the Lactococcus lactis diacetyl pathway. Lait 76:33-44.

Green, E.M., Boynton, Z.L., Harris, L.M., Rudolph, F.B., Papoutsakis, E.T. and Bennett, G.N. (1996) Genetic manipulation of acid formation pathways by gene inactivation in Clostridium Acetobutylicum ATCC 824. Microbiology 142:2079-2086.
Hansen, J. and Kiellandbrandt, M.C. (1996). Modification of biochemical pathways in industrial yeasts. Journal of Biotechnology 49:1-12.

Heinrich, R. and Rapoport, S.M. (1997). Metabolic regulation and mathematical models. In Progress in Biophysics and Molecular Biology, Vol. 32, Butler, J. A. V., Noble, D., Ed., Pergamon Press: Oxford, UK, pp 1-82.

Hershberger, C.L. (1996).Metabolic engineering of polyketide biosynthesis. Current Opinions in Biotechnology 7:560-2.

Holms, H. (1996). Flux analysis and control of the central metabolic pathways in Escherichia coli. FEMS Microbiology Reviews 19:85-116.

Ingram, L.O., Gomez, P.F., Lai, X., Moniruzzaman, M., Wood, B.E., Yomano, L.P. and York, S.W. (1998). Metabolic engineering of bacteria for ethanol production. Biotechnology \& Bioengineering 58:204-214.

Kacser, H. and Burns, J.A. (1973). The control of flux. Symposium of the Society of Experimental Biology 27:65104.

Keasling, J.D., Vandien, S.J. and Pramanik, J. (1998). Engineering polyphosphate metabolism in Escherichia coli - implications for bioremediation of inorganic contaminants. Biotechnology \& Bioengineering 58:231239.

Lessard, P. (1996). Metabolic engineering, the concept coalesces. Nature Biotechnology 14: 1654-1655.

Liao, J.C., Hou, S.Y. and Chao, Y.P. (1996a). Pathway analysis, engineering, and physiological considerations for redirecting central metabolism. Biotechnology \& Bioengineering 52:129-140.

Lu, J. L. and Liao, J.C. (1997). Metabolic engineering and control analysis for production of aromatics-role of transaldolase. Biotechnology \& Bioengineering 53:132137.

Mauch K., Arnold S. and Reuss M. (1997). Dynamic sensitivity analysis for metabolic systems. Chemical Engineering Science 52:2589-2598.

Misawa N.and Shimada H. (1998). Metabolic engineering for the production of carotenoids in non-carotenogenic bacteria and yeasts. Journal of Biotechnology 59:169-181.

Panke, S. and Sanchezromero, J. M. (1998). Engineering of quasi-natural Pseudomonas putida strains for toluene metabolism through an ortho-cleavage degradation 
pathway. Applied and Environmental Microbiology 64:748-751.

Park, S.M., Shawreid, C., Sinskey, A.J. and Stephanopoulos, G. (1997). Elucidation of anaplerotic pathways in Corynebacterium glutamicum via C-13-NMR spectroscopy and GC-MS. Applied Microbiology and Biotechnology 47:430-440.

Pines, O., Shemesh, S., Battat, E. and Goldber, I. (1997). Overexpression of cytosolic malate dehydrogenase (MDH2) causes overproduction of specific organic acids in Saccharomyces cerevisiae. Applied Microbiology and Biotechnology 48:248-255.

Poirier, Y., Nawrath, C. and Somerville, C. (1995). Production of polyhydroxyalkanoates, a family of biodegradable plastics and elastomers, in bacteria and plants. Biotechnology 13:142-150.

Prieto, M.A., Diaz, E. and Garcia, J.L. (1996). Molecular characterization of the 4-hydroxyphenylacetate catabolic pathway of Escherichia coli W - engineering a mobile aromatic degradative cluster. Journal of Bacteriology 178:111-120.

San, K.-Y., Bennett, G. N., Aristidou, A.A. and Chou, C.H .(1994). Strategies in high level expression of recombinant protein in Escherichia coli, Annals of the New York Academy of Sciences 721:257-267.

Savageau, M.A., Voit, E.O. and Irvine, D.H. (1987a). Biochemical systems theory and metabolic control theory: I. Fundamental similarities and differences. Mathematical Biosciences 86:127-145.

Savageau, M.A., Voit, E.O. and Irvine, D.H. (1987b). Biochemical systems theory and metabolic control theory: II. The role of summation and connectivity relationships. Mathematical Biosciences 86:147-169.

Shimada, H., Kondo, K., Fraser, P. D., Miura, Y., Saito, T and, Misawa, N. (1998). Increased carotenoid production by the food yeast Candida utilis through metabolic engineering of the isoprenoid pathway. Applied Microbiology and Biotechnology 64:2676-2680.

Schmidt, K., Marx, A., de Graaf, A. A., Wiechert, W., Sahm, H., Nielsen, J. and Villadsen, J. (1998). C-13 tracer experiments and metabolite balancing for metabolic flux analysis - comparing two approaches. Biotechnology and Bioengineering 58: 254-257.

Smirnoff, N. (1998). Plant resistance to environmental stress. Current Opinion in Biotechnology 9:214-219.
Small, J.R. and Kacser, H. (1993a). Responses of metabolic systems to large changes in enzyme activities and effectors. 1 The linear treatment of unbranched chains. European Journal of Biochemistry 213:613-624.

Small, J.R. and Kacser, H. (1993b). Responses of metabolic systems to large changes in enzyme activities and effectors. 2 The linear treatment of branched pathways and metabolite concentrations. Assessment of the general non-linear case. European Journal of Biochemistry 213:625-640.

Sprenger, G. A. (1996). Carbohydrate metabolism in Zymomonas mobilis - a catabolic highway with some scenic routes. FEMS Microbiology Letters 145:301-307.

Stassi, D.L., Kakavas, S.J., Reynolds, K.A., Gunawardana, G., Swanson, S., Zeidner, D., Jackson, M., Liu, H., Buko, A. and Katz, L. (1998). Ethyl-substituted erythromycin derivatives produced by directed metabolic engineering. Proceedings of the National Academy of Sciences USA 95:7305-7309.

Stephanopoulos, G. (1999). Metabolic fluxes and metabolic engineering, Metabolic Engineering 1:1-11.

Stephanopoulos, G. and Simpson, T.W. (1997). Flux amplification in complex metabolic networks. Chemical Engineering Science 52:2607-2627.

Stephanopoulos, G. and Sinskey, A. J. (1993). Metabolic engineering methodologies and future prospects. Trends in Biotechnology 11:392-396.

Stephanopoulos, G. and Vallino, J.J. (1991). Network rigidity and metabolic engineering in metabolite overproduction. Science 252:1675-1681.

Xu, B.W., Wild. J.R. and Kenerley, C.M. (1996). Enhanced expression of a bacterial gene for pesticide degradation in a common soil fungus. Journal of Fermentation \& Bioengineering 81:473-481.

Yang, Y.T., Bennett, G. N. and San, K.Y. (1998). Metabolic flux analysis of E. coli deficient in the acetate production pathway and expressing the $B$. subtilis acetolactate synthase. Metabolic Engineering. Accepted for publication. 